\title{
First catalogue of stars with photoelectric astrolabe in San Juan ${ }^{\star}$
}

\author{
Lu Lizhi $^{1}$, W.T. Manrique ${ }^{2}$, R. Perdomo ${ }^{3}$, R.C. Podesta ${ }^{2}$, Wang Zezhi ${ }^{1}$, E.L. Actis ${ }^{2}$, Zeng Fanmiao ${ }^{1}$, Zeng \\ Zhifang $^{1}$, E. Alonso ${ }^{2}$, A. Serafino ${ }^{2}$, Zhao Gang ${ }^{4}$ and J. Hormaechea ${ }^{3}$ \\ 1 Beijing Astronomical Observatory, Beijing, 100080, China \\ 2 Observatorio Astronomico Felix Aguilar, Benavidez 8175, San Juan, Argentina \\ 3 Observatorio Astronomico de La Plata, 1900-La Plata, Argentina \\ 4 Shanghai Astronomical Observatory, Shanghai, 200030, China
}

Received September 18; accepted October 20, 1995

\begin{abstract}
On the basis of data observed in San Juan of Argentina with the photoelectric astrolabe mark II (PA II) of Beijing Astronomical Observatory from Feb. 23, 1992 to Feb. 28, 1995, residuals of 7200 stars are reduced from about 230000 observations of stars. The mean precision of the residuals is $\pm 0.046^{\prime \prime}$. Using the data, the first catalogue of stars (CPASJ1) has been compiled. There are 2980 stars in this catalogue, including 989 FK5/FK4 Supp stars, 658 FK5 Ext stars, 387 SRS stars, 687 CAMC4 stars, 192 GC stars, and 72 Hipparcos stars. The mean precisions are $\pm 3.2 \mathrm{~ms}$ and $\pm 0.061^{\prime \prime}$ in right ascension and declination, respectively. The magnitudes of stars are from 2.0 to 11.3 . The declinations are from $-3^{\circ}$ to $-60^{\circ}$. The mean epoch is 1993.6 . Finally, systematic corrections of (CPASJ1-FK5) are given.
\end{abstract}

Key words: catalogue — astrometry — reference system

\section{Introduction}

The photoelectric astrolabes were made at Nanjing Astronomical Instrument Factory in 1974. These instruments are used for determinations of the astronomical time, latitude, and star positions as well as for research of astrogeodynamics.

The equal-altitude method gives good results not only for the determination of the Earth rotation parameters, but also in the improvement of catalogues. The potentiality of this method in the field of position astronomy has been shown by several general catalogues (Zhu et al. 1981; Working Group of GCPA, 1983; Working Group of CGSC, 1991; Lu 1991) of stars observed with the different marks of Chinese photoelectric astrolabes.

The project of Fundamental Reference System of Stars is important in the field of astrometry. Although the Hipparcos will make remarkable achievements, but there are many things to do after Hipparcos (Clauzet et al. 1990; Kovalevsky 1991), such as: the improvement of fundamental reference system of stars; the extension of fundamental catalogue to faint stars; the maintenance of the Hipparcos reference system; the extension of the Hipparcos catalogue. Two types of ground-based instruments could do

Send offprint requests to: Lu Lizhi

${ }^{\star}$ The catalogue is available in electronic form at the CDS via anonymous ftp 130.79.128.5 these works: the photoelectric meridian circles and photoelectric astrolabes, which both give star positions with a precision corresponding to a mean error of the order of $0.1^{\prime \prime}$.

During the IAU 20th General Assembly at Baltimore in 1988, the Working Group "Astrolabe", established by Commission 8 had decided to undertake the compilation of a New General Astrolabe Catalogue (NCGA). Astronomers between China and Argentina are interesting in the project. According to the cooperation between Beijing, San Juan, and La Plata Astronomical Observatories, the photoelectric astrolabe mark II of Beijing Astronomical Observatory was moved and installed at San Juan Observatory, Argentina in January 1992 for the observations of the catalogue of stars in the southern hemisphere. It is important for improvement of Fundamental Reference System of Stars.

\section{Instrument}

The photoelectric astrolabe Mark II No. 2 was installed at the Shahe Station of the Beijing Astronomical Observatory at the end of 1975 and was put into operation since Mar. 1976 (Luo 1979). The aperture of the object mirror is $200 \mathrm{~mm}$ and that of secondary mirror $49 \mathrm{~mm}$. The equivalent focal length is $2400 \mathrm{~mm}$. The zenith distance observed is $30^{\circ}$. Vacuum telescope tube and two reflecting 
Table 1. The relation between residual and magnitude

\begin{tabular}{|c|cccccccccc|}
\hline & & & & & & & & & & \\
$\bar{M}_{v}$ & 2.2 & 3.1 & 4.0 & 5.0 & 5.9 & 7.0 & 8.0 & 9.0 & 9.9 & 10.7 \\
$N$ & 32 & 122 & 260 & 507 & 751 & 588 & 705 & 870 & 551 & 115 \\
$\bar{V}_{\mathrm{m}}\left( \pm 0.01^{\prime \prime}\right)$ & -0.9 & -0.8 & 0.3 & 0.2 & 1.1 & 0.1 & 0.0 & 0.9 & 0.4 & 1.6 \\
$m_{\bar{V}_{\mathrm{m}}}\left( \pm 0.01^{\prime \prime}\right)$ & 1.1 & 0.6 & 0.4 & 0.3 & 0.3 & 0.5 & 0.6 & 0.5 & 0.7 & 1.6 \\
\hline
\end{tabular}

Table 2. The relation between residual and spectral type

\begin{tabular}{|c|cccccc|}
\hline Sp. & $\mathrm{B}$ & $\mathrm{A}$ & $\mathrm{F}$ & $\mathrm{G}$ & $\mathrm{K}$ & $\mathrm{M}$ \\
$N$ & 435 & 726 & 708 & 899 & 1543 & 164 \\
$\bar{V}_{\mathrm{s}}\left(0^{\prime \prime} .01^{\prime \prime}\right)$ & -0.8 & 0.1 & 0.7 & -0.2 & 0.9 & 1.1 \\
$m_{\bar{V}_{\mathrm{s}}}\left(0.01^{\prime \prime}\right)$ & 0.3 & 0.3 & 0.4 & 0.4 & 0.3 & 0.7 \\
\hline
\end{tabular}

Table 3. The group corrections $\mathrm{d} t, \mathrm{~d} \varphi$, and $\mathrm{d} z$

\begin{tabular}{|c|rrrrrrrrrrrr|r|}
\hline Group & 0 & 2 & 4 & 6 & 8 & 10 & 12 & 14 & 16 & 18 & 20 & $\begin{array}{r}22 \\
\text { closing } \\
\text { error }\end{array}$ \\
\hline $\mathrm{d} t\left(0.0001^{\mathrm{s}}\right)$ & & & & & & & & & & & & & -18 \\
$\sigma_{t}\left( \pm 0.0001^{\mathrm{s}}\right)$ & 10 & 10 & 10 & 10 & 10 & 10 & 10 & 10 & 11 & 11 & 11 & 11 & 36 \\
$\mathrm{~d} \varphi\left(0.001^{\prime \prime}\right)$ & 20 & 19 & -06 & -03 & 48 & -08 & -15 & 00 & 10 & 07 & -59 & -13 & -64 \\
$\sigma_{\mathrm{d} \varphi\left( \pm 0.001^{\prime \prime}\right)}(15$ & 14 & 14 & 13 & 13 & 13 & 14 & 14 & 15 & 16 & 16 & 15 & 50 \\
$\mathrm{~d} z\left(0.001^{\prime \prime}\right)$ & 04 & -28 & -68 & -35 & 25 & 05 & -14 & 36 & 41 & 18 & 08 & 07 & 82 \\
$\sigma_{\mathrm{d} z}\left( \pm 0.001^{\prime \prime}\right)$ & 11 & 11 & 11 & 11 & 11 & 11 & 10 & 11 & 11 & 11 & 12 & 12 & 38 \\
\hline
\end{tabular}

mirrors with a stable angle are used. This optical system of the astrolabe is quite stable.

Before modernization, the astrolabe was a semiautomatic instrument. So the instrument ought to be operated by the observer. It was hard work for 8-12 hours in one night, and the limiting magnitude was only 7.0.

Since 1987, the astronomers of Beijing Astronomical Observatory began to improve the instrument with two steps: the first step was to realize automatic observation; the second step was to realize photon-counting for increasing the limiting magnitude of observation. Keeping the original structure and optical system as far as possible, a simple and reliable scheme of automation was designed. The instrument is controlled with a microcomputer (PC/AT). Azimuth is measured with a synchronous inductor. A stepping motor is used for driving the instrument. The automatic observation with the instrument was accomplished in 1988.

A new method of photon-counting, so called "half widthadjusting", was developed and used for data processing instead of the original electric time recorder in 1990. After re-equipment, full automation was achieved with the calculation of the apparent position of stars, operating and tracking of the instrument, data acquisition and processing. The controlling software has a good in- terface between operator and computer. The operation is very easy. Even a new observer can use this astrolabe easily.

\section{The observation program}

The purpose of observation with PA II in San Juan is for improvement of the fundamental catalogue in southern hemisphere. The list of stars is selected from:

- a) FK5 fundamental stars (600 stars, 2 mag-8 mag)

- b) FK4 Supp stars (650 stars, 2 mag-8 mag)

- c) FK5 Ext stars (820 stars, $6 \mathrm{mag}-9.5 \mathrm{mag})$

- d) SRS stars (1300 stars, 6 mag-9.5 mag)

- e) IMF stars, that is Intermediate Fundamental Stars (1010 stars, 9 mag- $11.5 \mathrm{mag}$ )

- f) CAMC4 stars (1200 stars, 9 mag-11.5 mag)

- g) GC stars (350, 2 mag-6 mag)

- h) Radio stars (70 stars, 2 mag-11.5 mag)

There are about 6000 stars in the list. The magnitudes are from 2.0 to 11.5. The declinations are from $-3^{\circ}$ to $-60^{\circ}$, The positions of FK5, FK4 Supp, and FK5 Ext stars in J2000.0 are adopted from FK5 Fundamental Catalogue and FK5 Extend Fundamental Catalogue provided by CDS. The positions of Intermediate Fundamental Stars 
Table 4. a) The frequency distribution of $\Delta \alpha$

\begin{tabular}{|c|c|cccccc|}
\hline & $|\Delta \alpha|(\mathrm{ms})$ & $00-10$ & $10-20$ & $20-30$ & $30-40$ & $40-50$ & $>50$ \\
\hline \multirow{2}{*}{ FK5 } & $N$ & 338 & 106 & 21 & 1 & 1 & 0 \\
& $\%$ & 72.4 & 22.7 & 4.5 & 0.2 & 0.2 & 0.0 \\
\hline FK4 Supp & $N$ & 350 & 129 & 37 & 4 & 2 & 0 \\
& $\%$ & 67.0 & 24.7 & 7.1 & 0.8 & 0.4 & 0.0 \\
\hline FK5 Ext & $N$ & 306 & 213 & 93 & 27 & 9 & 10 \\
& $\%$ & 46.5 & 32.4 & 14.1 & 4.1 & 1.4 & 1.5 \\
\hline CAMC4 & $N$ & 333 & 196 & 90 & 32 & 14 & 16 \\
& $\%$ & 48.9 & 28.8 & 13.2 & 4.7 & 2.1 & 2.3 \\
\hline SRS & $N$ & 136 & 107 & 55 & 39 & 12 & 28 \\
& $\%$ & 35.1 & 27.6 & 14.2 & 10.1 & 3.1 & 7.2 \\
\hline
\end{tabular}

Table 4. b) The frequency distribution of $\Delta \delta$

\begin{tabular}{|c|c|cccccc|}
\hline & $|\Delta \delta|\left(0^{\prime \prime} .01\right)$ & $00-15$ & $15-30$ & $30-45$ & $45-60$ & $60-75$ & $>75$ \\
\hline FK5 & $N$ & 229 & 51 & 9 & 2 & 0 & 0 \\
& $\%$ & 78.7 & 17.5 & 3.1 & 0.7 & 0.0 & 0.0 \\
\hline FK4 Supp & $N$ & 199 & 90 & 22 & 4 & 1 & 0 \\
& $\%$ & 63.0 & 28.5 & 7.0 & 1.3 & 0.3 & 0.0 \\
\hline FK5 Ext & $N$ & 227 & 113 & 57 & 14 & 6 & 1 \\
& $\%$ & 54.3 & 27.0 & 13.6 & 3.3 & 1.4 & 0.2 \\
\hline CAMC4 & $N$ & 161 & 98 & 61 & 25 & 9 & 9 \\
& $\%$ & 44.4 & 27.0 & 16.8 & 6.9 & 2.5 & 2.5 \\
\hline SRS & $N$ & 114 & 73 & 47 & 23 & 10 & 6 \\
& $\%$ & 40.7 & 26.1 & 16.8 & 8.2 & 3.6 & 2.1 \\
\hline
\end{tabular}

Table 5. a) The systematic corrections (CPASJ1-FK5) $\Delta \alpha_{\alpha}$ and $\Delta \delta_{\alpha}$

\begin{tabular}{|c|rrrrrrrrrrrr|}
\hline$\alpha^{h}$ & 0 & 1 & 2 & 3 & 4 & 5 & 6 & 7 & 8 & 9 & 10 & 11 \\
\hline$\Delta \alpha_{\alpha}\left(0.0001^{\mathrm{s}}\right)$ & -16 & -32 & -46 & -35 & 5 & 53 & 78 & 66 & 29 & -4 & -13 & -2 \\
$\Delta \delta_{\alpha}\left(0.001^{\prime \prime}\right)$ & 25 & 24 & 13 & 1 & -2 & 7 & 26 & 42 & 47 & 36 & 13 & -11 \\
\hline \hline$\alpha^{h}$ & 12 & 13 & 14 & 15 & 16 & 17 & 18 & 19 & 20 & 21 & 22 & 23 \\
\hline$\Delta \alpha_{\alpha}\left(0.0001^{\mathrm{s}}\right)$ & 8 & 2 & -14 & -22 & -13 & 6 & 16 & 7 & -12 & -25 & -22 & -14 \\
$\Delta \delta_{\alpha}\left(0.001^{\prime \prime}\right)$ & -25 & -24 & -13 & -1 & 2 & -7 & -26 & -42 & -47 & -36 & -13 & 11 \\
\hline
\end{tabular}

Table 5. b) The systematic corrections (CPASJ1-FK5) $\Delta \alpha_{\delta}$ and $\Delta \delta_{\delta}$

\begin{tabular}{|c|c|c|c|c|c|c|c|c|c|c|c|c|}
\hline$\delta^{\circ}$ & -2.5 & -5.0 & -7.5 & -10.0 & -12.5 & -15.0 & -17.5 & -20.0 & -22.5 & -25.0 & -27.5 & -30.0 \\
\hline$\Delta \alpha_{\delta}\left(0.0001^{\mathrm{s}}\right)$ & 41 & 25 & 12 & 12 & 23 & 34 & 33 & 15 & -14 & -40 & -49 & -39 \\
\hline$\Delta \delta_{\delta}\left(0.001^{\prime \prime}\right)$ & -48 & -39 & -30 & -22 & -15 & -9 & -3 & 2 & 6 & 9 & & \\
\hline$\overline{\delta^{\circ}}$ & -32.5 & -35.0 & $\overline{-37.5}$ & -40.0 & -42.5 & $\overline{-45.0}$ & -47.5 & -50.0 & -52.5 & $\overline{-55.0}$ & -57.5 & \\
\hline$\Delta \alpha_{\delta}\left(0.0001^{\mathrm{s}}\right)$ & -14 & 12 & 24 & 13 & -19 & -57 & -81 & -70 & -19 & 25 & 50 & \\
\hline$\Delta \delta_{\delta}\left(0.001^{\prime \prime}\right)$ & & & & & & & 14 & 13 & 11 & 9 & 7 & \\
\hline
\end{tabular}


are provided by Dr. Thomas E. Corbin at U.S. Naval Observatory. The positions of radio stars are adopted from the literature (Walter 1990). The positions of GC stars are from the Chinese Geodetical Stars Catalogue, the others are from CAMC4 and SRS catalogues. All the positions of stars have been reduced to the epoch and equinox J2000.0.

\section{Reduction of the data}

\subsection{The reduction of star residuals}

The fundamental equation of observation is

$$
15 \cos \varphi_{0} \sin A X+\cos A Y-Z+\delta h=0,
$$

where

$\varphi_{0}$ - the adopted value of latitude at the site of the instrument;

$A$ - the azimuth of a star observed, measured eastwards from north;

$X$ - the observed clock correction;

$Y$ - the correction of latitude;

$Z$ - the correction of instrumental zenith distance;

$\delta h-$ a constant term which is related to the position of the instrument and systematic errors in the observation.

The residual of a star can be calculated from the formula:

$$
v=15 \cos \varphi_{0} \sin A X+\cos A Y-Z+\delta h .
$$

There are 384 stars in the fundamental groups. The corrections of astronomical time, latitude, zenith distance and residuals are obtained by the method of least squares. The residuals of the catalogue stars are calculated with the corrections of astronomical time, latitude and zenith distance of the reference group of stars. Then, the mean values of the residuals of stars are calculated by weighted means. The weights $\mathrm{P}$ are calculated from the formula:

$$
P=\frac{0.1}{\sigma^{2}}
$$

where $\sigma$ is the precision of a single star in the reference group of stars.

\subsection{The reduction of position corrections of stars}

Assumed $V_{\mathrm{e}}$ and $V_{\mathrm{w}}$ are the residuals reduced to the mean instrumental system and considering the instrumental system errors at both eastern and western transits, we obtain the equation defining the position corrections ( $\mathrm{Lu}$ et al. 1980):

$$
\Delta \alpha=\frac{V_{\mathrm{e}}-V_{\mathrm{w}}}{30 \cos \varphi_{0}|\sin A|}+\xi
$$

and

$$
\Delta \delta=-\frac{V_{\mathrm{e}}+V_{\mathrm{w}}-2 K}{2 \cos q}+l \cos \delta
$$

where $q$ - the parallactic angle of a star as it transits the almucantar of the astrolabe.

$2 K$ can be calculated from

$$
K=\frac{1}{2}\left(V_{\mathrm{e}}+V_{\mathrm{w}}\right)_{q=90^{\circ}}
$$

\section{Results}

From Feb. 23, 1992 to Feb. 28, 1995, there are about 230000 observations of stars observed on 870 days. From these data, 7200 residuals are obtained with the mean precision of $\pm 0.046^{\prime \prime} ; 2980$ position corrections of stars have been reduced by Eqs. (4) and (5) from double transits of given stars at both eastern and western passages with average precision $\pm 3.2 \mathrm{~ms}$ and $\pm 0.061^{\prime \prime}$ in right ascension and declination, respectively. The unknown terms $\xi$ and $l \cos \delta$ included in the calculated $\Delta \alpha$ and $\Delta \delta$ are neglected. But $\Delta \delta$ for the stars with $|\cos q|<0.3$ is not given.

\subsection{The relation between residual and magnitude}

The mean values of residuals of stars by weighted mean according to the precision of residuals and magnitudes are given in Table 1 . In Table $1, \bar{M}_{v}$ is the mean values of observed magnitudes, $N$ the number of stars observed, $\bar{V}_{\mathrm{m}}$ and $m_{\bar{V}_{\mathrm{m}}}$ the mean value of residuals and its accuracy.

\subsection{The relation between residual and spectral types}

The mean values of residuals of stars by weighted mean according to the precision of residuals and spectral type are given in Table 2, where $N$ is the number of stars observed, $\bar{V}_{\mathrm{s}}$ and $m_{\bar{V}_{\mathrm{s}}}$ the mean value of residuals and its accuracy.

From Tables 1 and 2, one sees that the instrumental errors on magnitude and spectral type are very small.

\subsection{The calculation of $2 K$}

Strictly speaking, the calculation of $2 K$ should be made using the stars with $\cos q=0$, that is $q=90^{\circ}$. But these stars are very few. So we used the stars with $|\cos q|<0.2$ (in this catalogue there are 490 such stars) to calculate $2 K$. The weighted mean value:

$$
2 K=0.020^{\prime \prime} \pm 0.003^{\prime \prime} .
$$

\subsection{The group corrections}

With the results of PA II from Feb. 1992 to Feb. 1995, the group corrections of $\mathrm{d} t, \mathrm{~d} \varphi$ and $\mathrm{d} z$ are calculated with the chain method. The results are given in Table 3.

From Table 3, we see that the closing errors of $\mathrm{d} z$ is very small. 


\subsection{The frequency distributions of $\Delta \alpha$ and $\Delta \delta$}

The frequency distribution of $\Delta \alpha$ 's and $\Delta \delta$ 's are given in Tables $4 \mathrm{a}$ and $4 \mathrm{~b}$ respectively.

From Tables $4 \mathrm{a}$ and $4 \mathrm{~b}$, we can see that the position corrections of about $95 \%$ of FK5 stars are less than $20 \mathrm{~ms}$ in $\Delta \alpha$ or $0.30^{\prime \prime}$ in $\Delta \delta$.

\subsection{The systematic corrections}

By the analytic method (Bien 1979; Working Group of GCPA, 1983) and adopting the $\Delta \alpha$ and $\Delta \delta$ of FK5/FK4 Supp stars obtained from the double transits, the systematic corrections of the catalogue of stars (CPASJ1-FK5) are analyzed. The systematic corrections on the right ascension and declination, $\Delta \alpha_{\alpha}, \Delta \delta_{\alpha}, \Delta \alpha_{\delta}, \Delta \delta_{\delta}$ are given in Tables $5 \mathrm{a}$ and $5 \mathrm{~b}$, respectively.

\section{The description of the catalogue}

The catalogue (CPASJ1) will be available in the CDS. In the catalogue, the description of each column is given as follows:

1: Number of CPASJ1;

2: Number of FK5 (recorder: 1 - 467), FK4 Supp (recorder: 468 - 989), FK5 Ext (recorder: 990 - 1647), SRS (recorder: 1648 - 2034), CAMC4 (recorder: 2035 -2715), GC (recorder 2716 - 2908) and Hipparcos (recorder: 2909 - 2980), respectively.

3: Visual magnitude;

4: Spectral type;

5: Position in right ascension of reference catalogue for epoch and equinox J2000.0;

6: Position in declination of reference catalogue for epoch and equinox J2000.0;

7: Position correction of right ascension in units of $0.001^{\mathrm{s}}$; 8: Mean error of the correction on right ascension in units of $0.001^{\mathrm{s}}$;

9: Position correction of declination in units of $0.01^{\prime \prime}$;

10: Mean error of the corrections in declination in units of $0.01^{\prime \prime}$;

11: Number of observations in the eastern transit;

12: Number of observations in the western transit;

13: Mean epoch of observations.

Acknowledgements. The authors wish to thank the CONICET of Argentina, Jan Juan and La Plata Universities, the State Sciences and Technology Commission of China, Chinese Academy of Sciences, National Natural Science Foundation of China, Chinese Astronomy Commission for their support for the project.

\section{References}

Carlsberg Meridian Catalogue in La Palma, 1989

Bien R., Veroff, 1978, Astron. Rechen-Institut, Heidelberg, No. 29

Clauzet L.B.F., Benevides Soares P., et al., 1990, Guidelines for the compilation of a New Astrolabe Catalogue

Kovalevsky J., 1991, Astrophys. Space Sci. 177, 457

Lu Lizhi, 1991, Publ. Beijing Astron. Obs. 17, 1

Luo Dingjiang, et al., 1979, Publ. Beijing Astron. Obs. 1, 56

Walter H.G., et al., 1990, A\&AS 86, 357

Working Group of GCPA, 1983, Acta Astron. Sin. 24, 267

Working Group of CGSC, 1991, Publ. House of Surveying and Mapping, Beijing

Zhu Yuanxian, Lu Lizhi, Luo Dingjiang, Ling Zhaofen, 1981, Publ. Beijing Astron. Obs. 1, 1

Lu Lizhi, Luo Dingjiang, et al., 1980, Acta Astron. Sin. 22, 305 